\title{
Article \\ Changes in Stress-Mediated Markers in a Human Cardiomyocyte Cell Line under Hyperglycemia
}

\author{
Vikram Thakur ${ }^{1}$, Narah Alcoreza ${ }^{2}$, Jasmine Cazares ${ }^{2}$ and Munmun Chattopadhyay ${ }^{1,2, *(D)}$ \\ 1 Center of Emphasis in Diabetes and Metabolism, Department of Molecular and Translational Medicine, \\ Paul L. Foster School of Medicine, Texas Tech University Health Sciences Center El Paso, \\ El Paso, TX 79905, USA; vikram.thakur@ttuhsc.edu \\ 2 Graduate School of Biomedical Sciences, Texas Tech University Health Sciences Center El Paso, \\ El Paso, TX 79905, USA; nalcorez@gmail.com (N.A.); jascaz05@gmail.com (J.C.) \\ * Correspondence: munmun.chattopadhyay@ttuhsc.edu; Tel.: +1-(915)-215-4170; Fax: +1-(915)-215-8875
}

Citation: Thakur, V.; Alcoreza, N.; Cazares, J.; Chattopadhyay, $\mathrm{M}$. Changes in Stress-Mediated Markers in a Human Cardiomyocyte Cell Line under Hyperglycemia. Int. J. Mol. Sci. 2021, 22, 10802. https://doi.org/ $10.3390 /$ ijms221910802

Academic Editors: Raffaele Marfella and Maria Luisa Balestrieri

Received: 8 September 2021

Accepted: 1 October 2021

Published: 7 October 2021

Publisher's Note: MDPI stays neutral with regard to jurisdictional claims in published maps and institutional affiliations.

Abstract: Diabetes is a major risk factor for cardiovascular diseases, especially cardiomyopathy, a condition in which the smooth muscles of the heart become thick and rigid, affecting the functioning of cardiomyocytes, the contractile cells of the heart. Uncontrolled elevated glucose levels over time can result in oxidative stress, which could lead to inflammation and altered epigenetic mechanisms. In the current study, we investigated whether hyperglycemia can modify cardiac function by directly affecting these changes in cardiomyocytes. To evaluate the adverse effect of high glucose, we measured the levels of gap junction protein, connexin 43 , which is responsible for modulating cardiac electric activities and Troponin I, a part of the troponin complex in the heart muscles, commonly used as cardiac markers of ischemic heart disease. AC16 human cardiomyocyte cells were used in this study. Under hyperglycemic conditions, these cells demonstrated altered levels of connexin 43 and Troponin-I after $24 \mathrm{~h}$ of exposure. We also examined hyperglycemia induced changes in epigenetic markers: H3K9me1, Sirtuin-1 (SIRT1), and histone deacetylase (HDAC)-2 as well as in inflammatory and stress-related mediators, such as heat shock protein (HSP)-60, receptor for advanced glycation end products (RAGE), toll-like receptor (TLR)-4, high mobility group box (HMGB)-1 and CXC chemokine receptor (CXCR)-4. Cardiomyocytes exposed to $25 \mathrm{mM}$ glucose resulted in the downregulation of HSP60 and SIRT1 after $48 \mathrm{~h}$. We further examined that hyperglycemia mediated the decrease in the gap junction protein CX43, as well as CXC chemokine receptor CXCR4 which may affect the physiological functions of the cardiomyocytes when exposed to high glucose for 24 and $48 \mathrm{~h}$ Upregulated expression of DNA-binding nuclear protein HMGB1, along with changes in histone methylation marker $\mathrm{H} 3 \mathrm{~K} 9$ me1 have demonstrated hyperglycemia-induced damage to cardiomyocyte at $24 \mathrm{~h}$ of exposure. Our study established that 24 to $48 \mathrm{~h}$ of hyperglycemic exposure could stimulate stress-mediated inflammatory mediators in cardiomyocytes in vitro. These stress-related changes in hyperglycemia-induced cardiomyocytes may further initiate an increase in injury markers which eventually could alter the epigenetic processes. Therefore, epigenetic and inflammatory mechanisms in conjunction with alterations in a downstream signaling pathway could have a direct effect on the functionality of the cardiomyocytes exposed to high glucose during short and long-term exposures.

Keywords: hyperglycemia; cardiomyocyte; Connexin 43; inflammation; oxidative stress

\section{Introduction}

Diabetes mellitus is a disease characterized by the body's inability to produce or properly respond to insulin which ultimately causes elevated blood glucose levels. Over time, high levels of blood glucose lead to micro- and macro-vascular damage to various organs of both Types 1 and 2 diabetic patients. Cardiomyopathy is one of the most lifethreatening macro-vascular complications of the diabetes. Cardiomyopathy is defined as progressive heart disease that affects the heart muscle due to thickening, rigidity or enlargement of the muscles resulting in a dysfunctional heart [1]. As the heart grows 
weaker, patients may get afflicted with worsening complications such as heart failure, arrhythmias, or valve problems [1]. The human heart is made of various cells including cardiomyocytes (CMs), fibroblasts (FBs), endothelial cells (ECs), perivascular cells, smooth muscle cells and neuronal cells. However, approximately $70-85 \%$ of the human heart is composed of CMs [2]. In the current study, we examined the early signs of stress in CMs exposed to hyperglycemic conditions that may lead to alterations in cardiomyocyte signaling, changes in injury markers and downstream indicators of oxidative stress, as well as epigenetic modifications and alterations in inflammatory mediators.

Connexin 43 (Cx43) is a transmembrane gap junction protein located between adjacent cells that functions to coordinate the depolarization of cardiac muscle during contractions of the heart. Chronic hyperglycemic condition in diabetes may disrupt and change the intercellular communication due to alterations in Cx43. Troponins are a group of complex proteins that regulate muscular contraction in cardiac and skeletal muscles and used as a marker to detect cardiac damage. Troponins consist of three subunits (C, T, and I), localized in the myofibrils. Troponin I is a cardiac specific inhibitory isotype that determines myocardial impairment [3]. Hyperglycemic condition initiates reduced mitochondrial enzyme activity and an increase in reactive oxygen species (ROS) formation. Hyperglycemia-mediated rise in oxidative stress is also linked with increased advanced glycation end products (AGEs) formation along with the enhanced expression of the receptor for AGEs (RAGE). Increased RAGE expression causes activation of various cellular pathways along with release of high mobility group box 1 (HMGB1) that bind to RAGE and toll-like receptor 4 (TLR4) with high affinity eventually stimulating a host of proinflammatory events [4]. TLR4 is transmembrane pattern recognition receptor (PRR) family of proteins [5]. Previous studies have demonstrated that high glucose induces increase in TLR4 expression in cardiac cells in a dose- and time-dependent manner [6]. Heat shock proteins (HSP) are chaperone proteins that regulate intracellular signaling. Earlier studies have suggested the cardiac protective role of HSP60 in cardiomyocytes, and reduced expression of HSP60 under chronic hyperglycemic condition may contribute to the progression of diabetic cardiomyopathy [7-9]. Epigenetic mechanisms contribute to the continuous and potential molecular modifications including DNA methylation and histone modifications under hyperglycemic condition that may cause aberrant and long-lasting changes in cardiomyocytes pathophysiology. Histone 3 lysine 9 mono methylation (H3K9me1), a marker for post-translational histone modification has appeared to have a prospective role in the progression of cardiac complications in diabetes [10]. Histone methylation may lead to inflammation and damage to the cardiac cells, leading to cardiomyopathy [2]. High glucose may affect specific changes in histone marks at the promoter sites of genes encoding inflammatory cytokines in cardiomyocyte cell lines [11]. Dysregulation of epigenetic posttranscriptional modifications of histones in chromatin is thought to be associated with the pathology of many disease models, including CVD. Balance of histone acetyltransferases (HATs) and deacetylases (HDACs) control the histone lysine acetylation. HDAC activity also play a crucial role in the severity of the cardiac damage with respect to myocardial remodeling. HDAC2 expression is altered in hyperglycemic condition [12]. Sirtuin (SIRT) 1, a nicotinamide adenine dinucleotide $(\mathrm{NAD})^{+}$-dependent class III histone deacetylase, has been reported to play a significant role in cellular metabolism, longevity as well as support against DNA damage $[13,14]$. In presence of stress, maintenance of $\mathrm{NAD}^{+}$is challenged which could affect cellular functions such as insulin secretion and signaling [15]. SIRT1 has been linked with insulin sensitivity and glucose homeostasis and studies have shown that patients with diabetes had an $80 \%$ reduction in SIRT1 levels. The present study describes that increased oxidative stress induced by hyperglycemia on CMs could lead to altered levels of inflammatory and epigenetic markers which may ultimately cause cardiac dysfunction in patients with diabetes. 


\section{Results}

\subsection{High Glucose Insult Results in Alterations in Connexin 43 and Troponin I Expression}

Connexin $43(\mathrm{C} \times 43)$ is a gap junction protein that maintains normal heart functions by regulating ventricular contractions, though under stress the functionality is altered. Cx43 expression under hyperglycemic conditions $(25 \mathrm{mM})$ exhibited significant down regulation at 24-h exposure. These observations were further confirmed via immunocytochemistry studies, where $\mathrm{C} \times 43$ expression was significantly downregulated in the presence of elevated glucose levels at $24 \mathrm{~h}$ on two separate experiments (Figure 1a,b). These findings were further analyzed for Troponin I (Trp-I) expression after $24 \mathrm{~h}$ hyperglycemic exposure to cardiomyocytes. Troponin I is required to regulate the contractions of the heart and skeletal muscles and upregulation of Trp-I demonstrates damage of the cardiomyocytes or heart muscles. Our study indicated a visible upregulation of Troponin I expression in $\mathrm{CM}$ cells exposed to the hyperglycemic conditions, when compared to the normo-glycemic condition (Figure 2).

a.

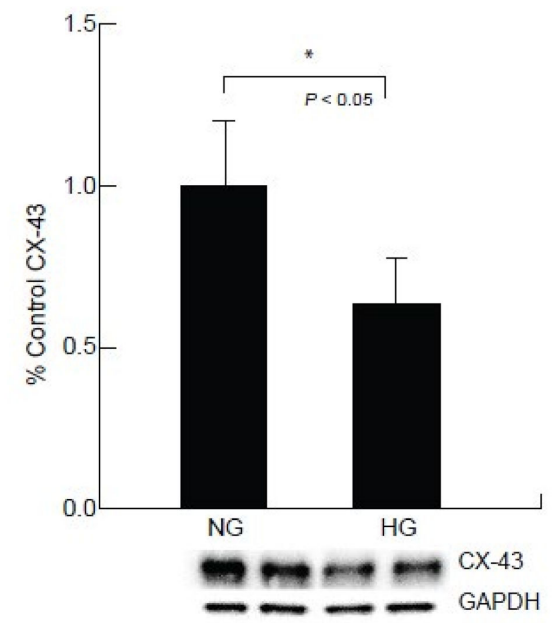

b.
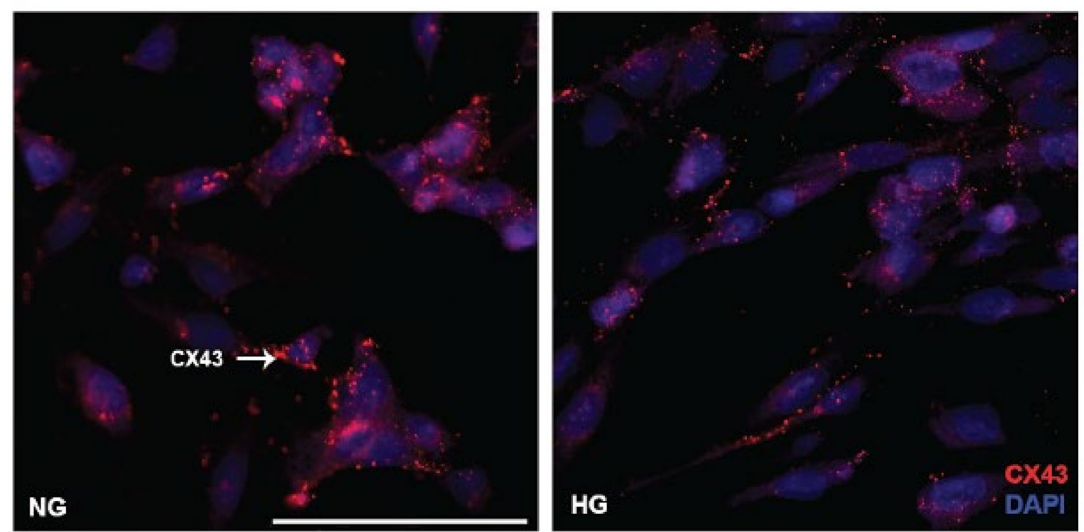

Figure 1. Hyperglycemic condition results in alterations in Connexin 43 expression in cardiomyocytes. Gap junction protein connexin 43 (Cx43) expression was assessed by Western blot analysis as well as by immunohistochemistry in cardiomycytes. (a). Western blot analysis demonstrated a decrease in CX43 expression in the hyperglycemia exposed cardiomyocytes compared to the normoglycemic condition $\left(\mathrm{NG},{ }^{*} p<0.05\right)$. (b). Immunocytochemical analysis also exhibited reduced $\mathrm{CX} 43$ expression (red) in CM under hyperglycemic condition compared to cardiomyocytes under normal glucose condition after $24 \mathrm{~h}$ of exposure. (Scale bar $=100 \mu \mathrm{m}$ ). 
a.

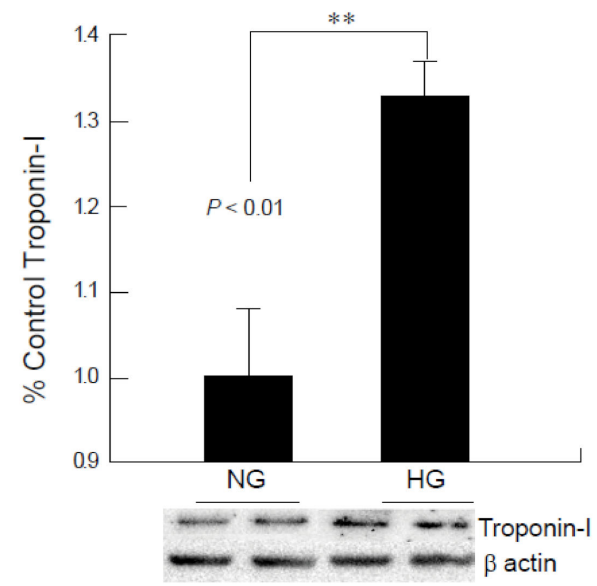

b.

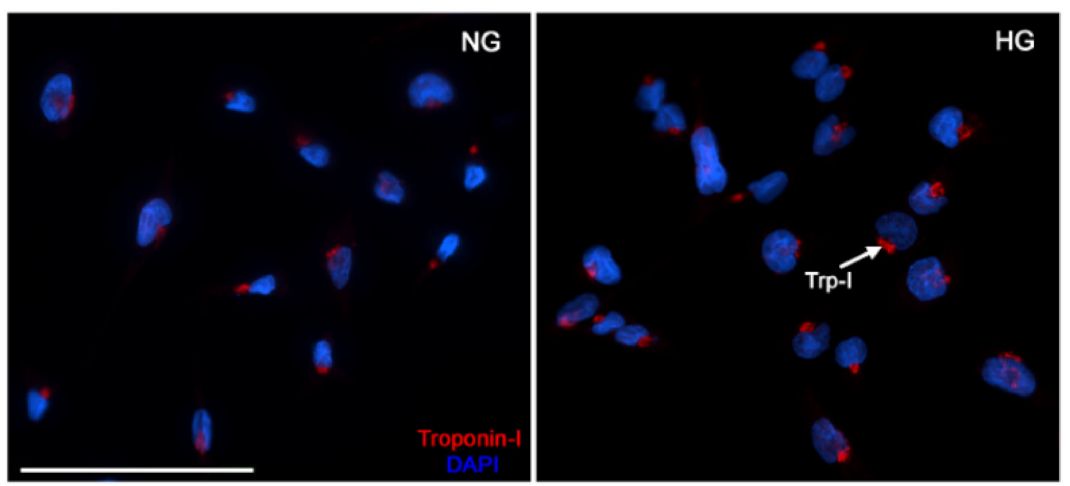

Figure 2. Hyperglycemia mediates increased Troponin I expression in the cardiomyocytes. (a) Western blot analysis of AC16 cardiomyocytes given hyperglycemic insult for $24 \mathrm{~h}$ showed an increase in the expression of Trp-I as compared to cells exposed to normal glucose (NG; ${ }^{* *} p<0.01$ ). (b) Immunocytochemical analysis of cardiomyocytes exposed to high glucose (HG) for $24 \mathrm{~h}$ showed an increase in the expression of Troponin I (red). (Scale bar $=100 \mu \mathrm{m})$.

\subsection{Hyperglycemia Leads to Altered Signaling of HSP60}

Heat shock protein 60 (HSP60) is a chaperone protein member of the HSP family which typically functions in mitochondria as well as cytosol, membrane and extracellular space, under stress, inflammation and immune mediated or other cellular events. Western blot analysis of HSP60 showed a downregulation of protein ability after a 48-h exposure to hyperglycemia, indicating that mechanisms may be altered as a result of the chronic stress (Figure 3). These findings were confirmed via immunocytochemistry studies, where a decrease in HSP60 was noted in the high glucose conditions as compared to the normal glucose conditions. Further analysis unveiled that heat shock protein 70 (HSP70), involved in inhibition of aggregation by binding to unfolded proteins by preventing further cellular damage, was significantly downregulated $\left({ }^{*} p<0.05\right)$ after 24 and $48 \mathrm{~h}$ of exposure to an elevated glucose ( $25 \mathrm{mM})$ environment (Figure S1).

\subsection{Hyperglycemia Induces Elevated Expression of HMGB1 and H3K9me1 in Cardiomyocyte}

HMGB1 is a non-chromosomal DNA-binding protein, which may release from cardiomyocytes under certain physiological conditions in response to stress. The immunocytochemistry and Western blot studies demonstrated an upregulation of HMGB1 (Figure 4a,b) along with its receptors RAGE and TLR4 (Figure 5a,b), that may lead to damage of CM cells as indicated by the upregulation of other stress-induced markers after $24 \mathrm{~h}$ of hyperglycemic exposure. 


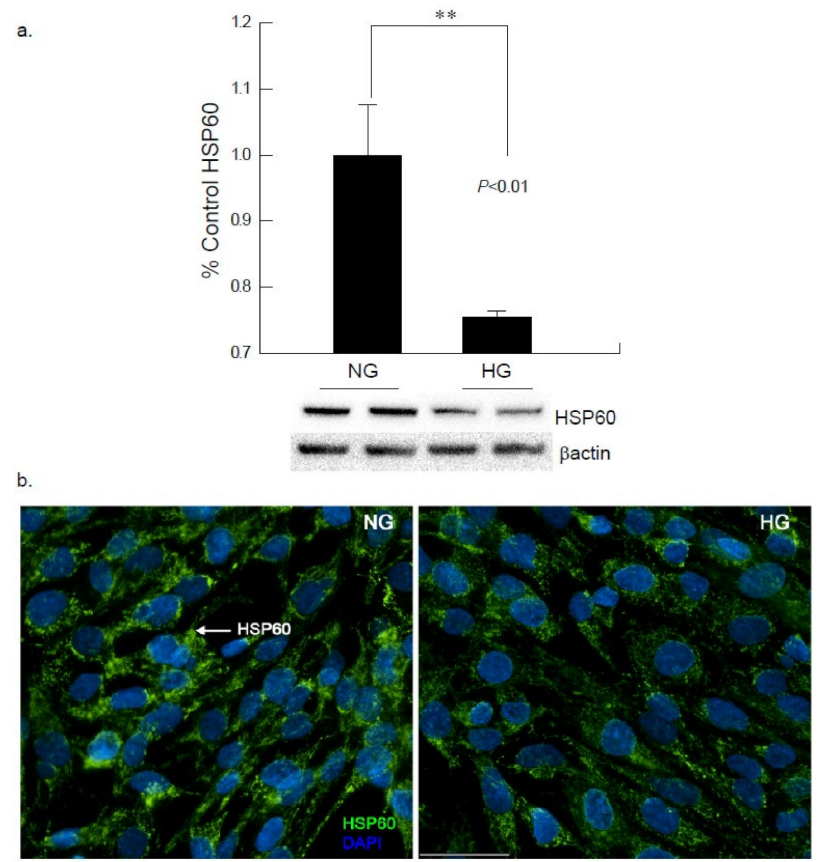

Figure 3. Hyperglycemic insult in cardiomyocytes reduces HSP60 expression. (a) Western blot analysis of AC16 cardiomyocytes following hyperglycemic insult for $48 \mathrm{~h}$ showed a decrease in the expression of HSP60 as compared to cells exposed to normal glucose (NG; $\left.{ }^{* *} p<0.01\right)$. (b) Immunocytochemical analysis of cardiomyocytes exposed to high glucose (HG) for $48 \mathrm{~h}$ also confirmed the decrease in the expression of HSP60 (green). (Scale bar $=50 \mu \mathrm{m}$ ).
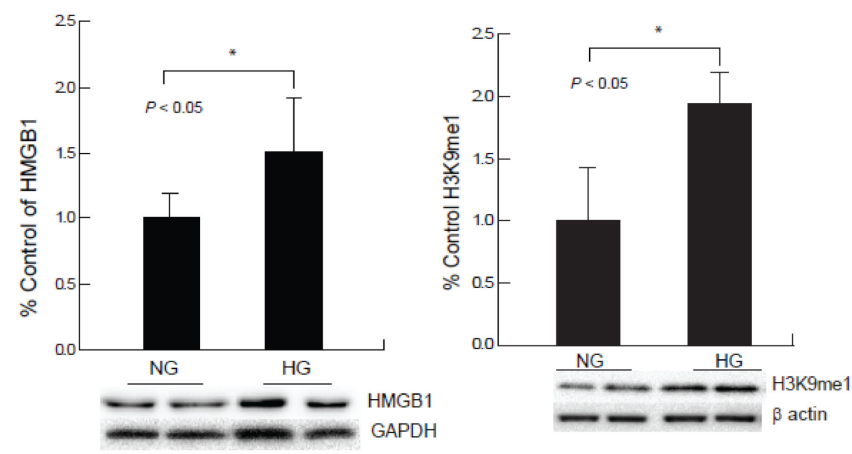

b.

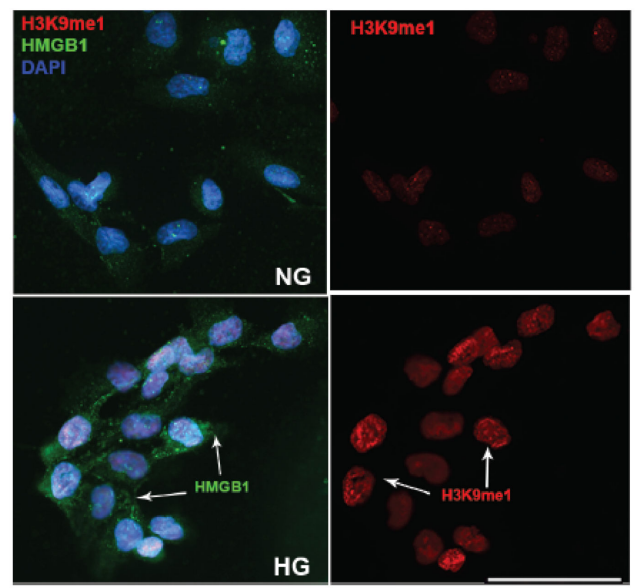

Figure 4. Hyperglycemia induces elevated expression of HMGB1 and H3K9me1 in cardiomyocyte. 
AC-16 cardiomyocytes revealed that stress mediated high glucose exposure increased expression of histone 3 lysine 9 mono-methylation (H3K9me1) as well as inflammatory mediator HMGB1 (high mobility group box 1). (a) Western blot analysis showed an increase in HMGB1 after $24 \mathrm{~h}$ of exposure to high glucose (HG) in comparison to normal glucose conditions (NG; ${ }^{*} p<0.05$ ). (b) Immunocytochemical analysis of cardiomyocytes exposed to high glucose for $24 \mathrm{~h}$ showed an increase in the expression of H3K9me1 (red) along with increased inflammatory mediator HMGB1 (green). (Scale bar $=100 \mu \mathrm{m}$ ). (c) Western blot analysis showed an increase in H3K9me1 after $24 \mathrm{~h}$ of exposure to high glucose in comparison to normal glucose conditions (NG; ${ }^{*} p<0.05$ ).

a

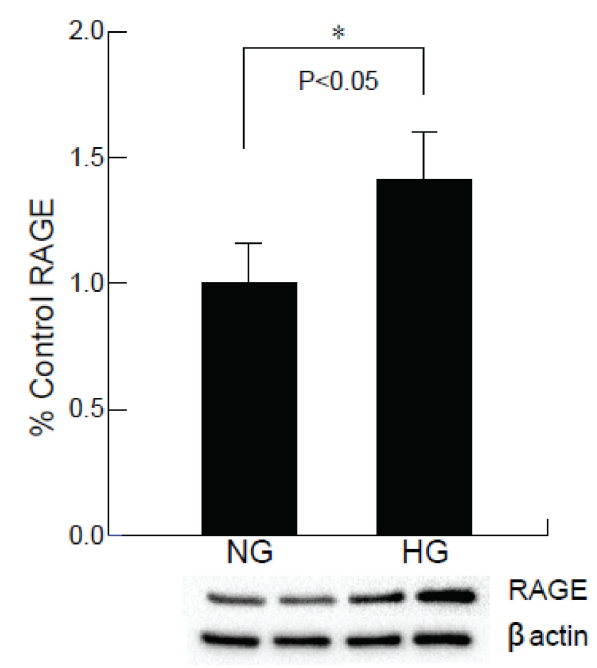

b.

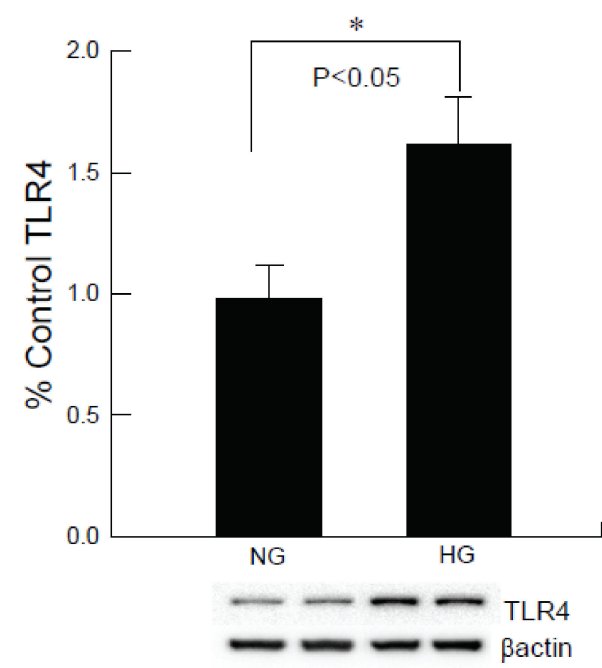

Figure 5. Hyperglycemic exposure exhibits altered levels of RAGE and TLR4 in AC-16 cardiomyocytes. (a) Increased RAGE expression was observed under the hyperglycemic condition in cardiomyocytes compared to normo-glycemic condition $\left({ }^{*} p<0.05\right)$. (b) Western blot analysis of AC16 cardiomyocytes under hyperglycemic conditions for $24 \mathrm{~h}$ showed a statistically significant increase in the expression of TLR4 as compared to cells exposed to normal glucose $\left({ }^{*} p<0.05\right)$.

Epigenetic modifications play critical part in cardiac dysfunction and not many studies demonstrated the role of epigenetic processes and cardiac dysfunction under hyperglycemic milieu. High glucose conditions may modify gene transcription by changing the post-translational modifications of histone protein. Our study showed a significant increase of histone mono-methylation at lysine 9 (H3K9me1) in CM cells in the presence of high glucose (Figure 4b,c) as demonstrated by immunocytochemistry and Western blot studies.

\subsection{AC-16 Cells under Hyperglycemic Conditions Upregulates RAGE and TLR4 Expression}

Following 24-h exposure to high glucose, the cardiomyocytes exhibited an increase in oxidative stress marker, receptor for advanced glycation end products (RAGE) and inflammatory mediator toll-like receptor 4 (TLR4) expression as evaluated by western blot analysis (Figure 5a,b). The RAGE is expressed in the cardiomyocytes, and an increase in RAGE expression suggests injuries to the CM cells due to hyperglycemic condition. Both RAGE and TLR4 bind to its ligand HMGB1.

\subsection{Hyperglycemia Modifies Chemokine Receptor CXCR4 Expression in Cardiomyocytes}

To understand the underlying mechanisms and the role of inflammatory mediators in cardiac impairment, the CXC chemokine receptor 4 (CXCR4), a G protein-coupled receptor (GPCR) was evaluated. Western blot analysis of the cardiomyocytes demonstrated a significant decrease in the CXCR4 expression at $48 \mathrm{~h}$ of exposure under hyperglycemic condition compared to the normo-glycemic condition (Figure 6), which further confirmed its possible role in prolonged inflammation. The decrease in the CXCR4 expression by long-term exposure to hyperglycemia induces damage to CM cells. 
a.
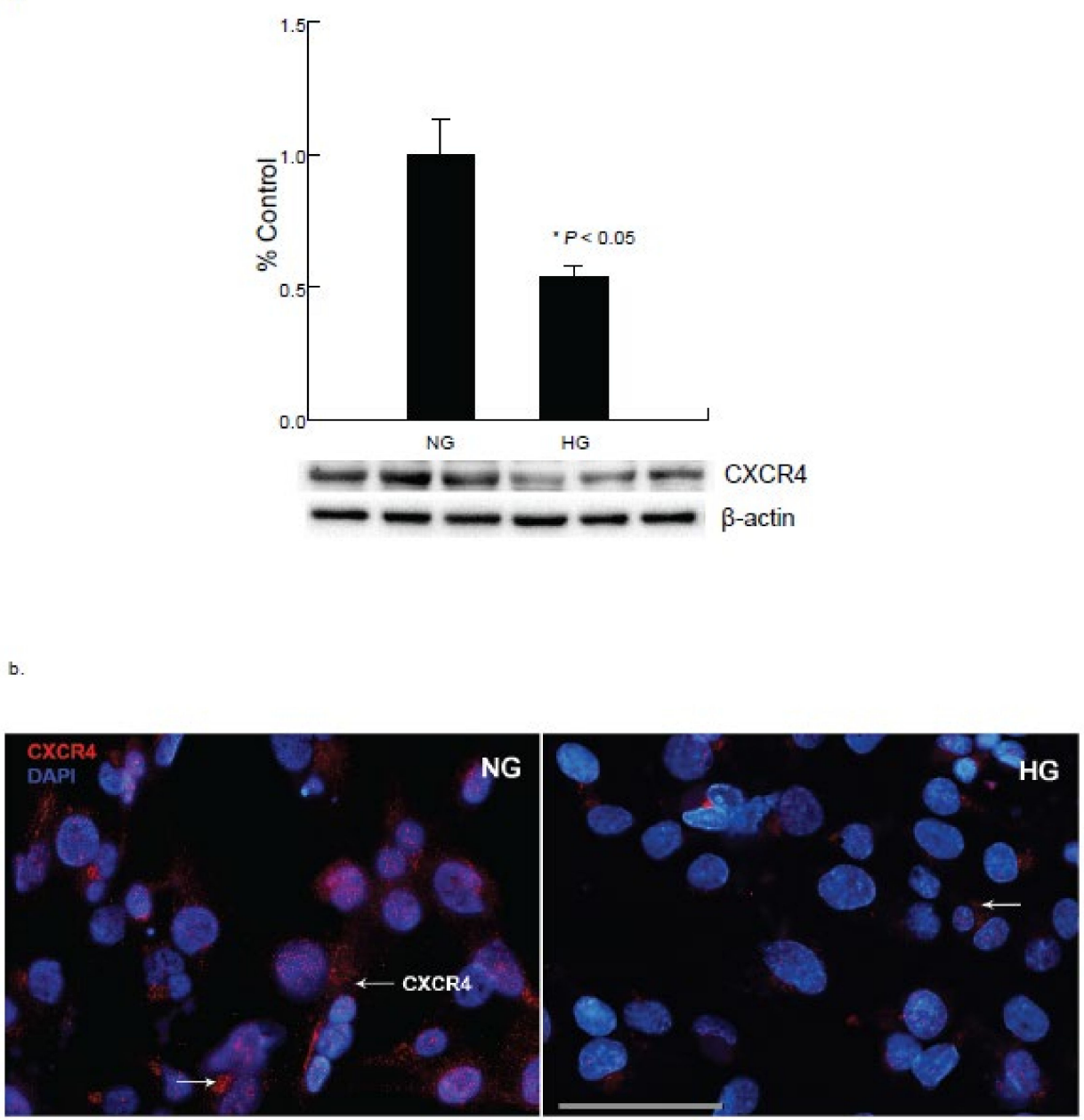

Figure 6. Hyperglycemia alters chemokine receptor CXCR4 expression in cardiomyocytes. (a) Western blot analysis of cardiomyocytes exposed to hyperglycemic condition for $48 \mathrm{~h}$ showed reduction in the expression of CXCR4 (* $p<0.05)$. (b) Immunocytochemical analysis of cardiomyocytes exposed to high glucose (HG) for $48 \mathrm{~h}$ showed decrease in the expression of CXCR4 (red). (Scale bar $=100 \mu \mathrm{m}$ ).

\subsection{Hyperglycemia Leads to Increased HDAC2 Expression in Cardiomyocyte}

To investigate the potential role of epigenetic modifications of class I histone deacetylases in cardiac cells under hyperglycemic condition, we analyzed the expression of HDAC2 in AC- 16 cells that are exposed to $25 \mathrm{mM}$ of glucose for 24 and $48 \mathrm{~h}$. Western blot and immunocytochemistry were performed to analyze the expression of HDAC2 in cardiomyocytes under normoglycemic and hyperglycemic conditions. The results indicated that the protein levels of HDAC2 were markedly elevated in hyperglycemic cardiomyocytes compared the normoglycemic cardiomyocytes after $24 \mathrm{~h}$ of high glucose exposure (Figure $7 \mathrm{a}, \mathrm{b}$ ).

\subsection{Hyperglycemic Condition Downregulates Sirtuin-1 Expression in CM}

To define whether physiologically-relevant hyperglycemic conditions could further affect class III histone deacetylase, SIRT1 expression, AC16 CM cells were exposed to high glucose $(25 \mathrm{mM})$ media; $48 \mathrm{~h}$ of exposure exhibited a decreased expression in SIRT1 levels. SIRT1 is an NAD+ dependent deacetylase which is important for metabolic control; when exposed to hyperglycemic induced stress, a shift in the NADH/NAD+ratio has been observed. Given the possible association between hyperglycemia and inflammation, the results confirm an inverse relationship between SIRT1 expression and hyperglycemic condition. Following a 48-h incubation period in elevated glucose, a significant decrease $(* * *<0.001)$ of SIRT1 was noted (Figure 7c). 

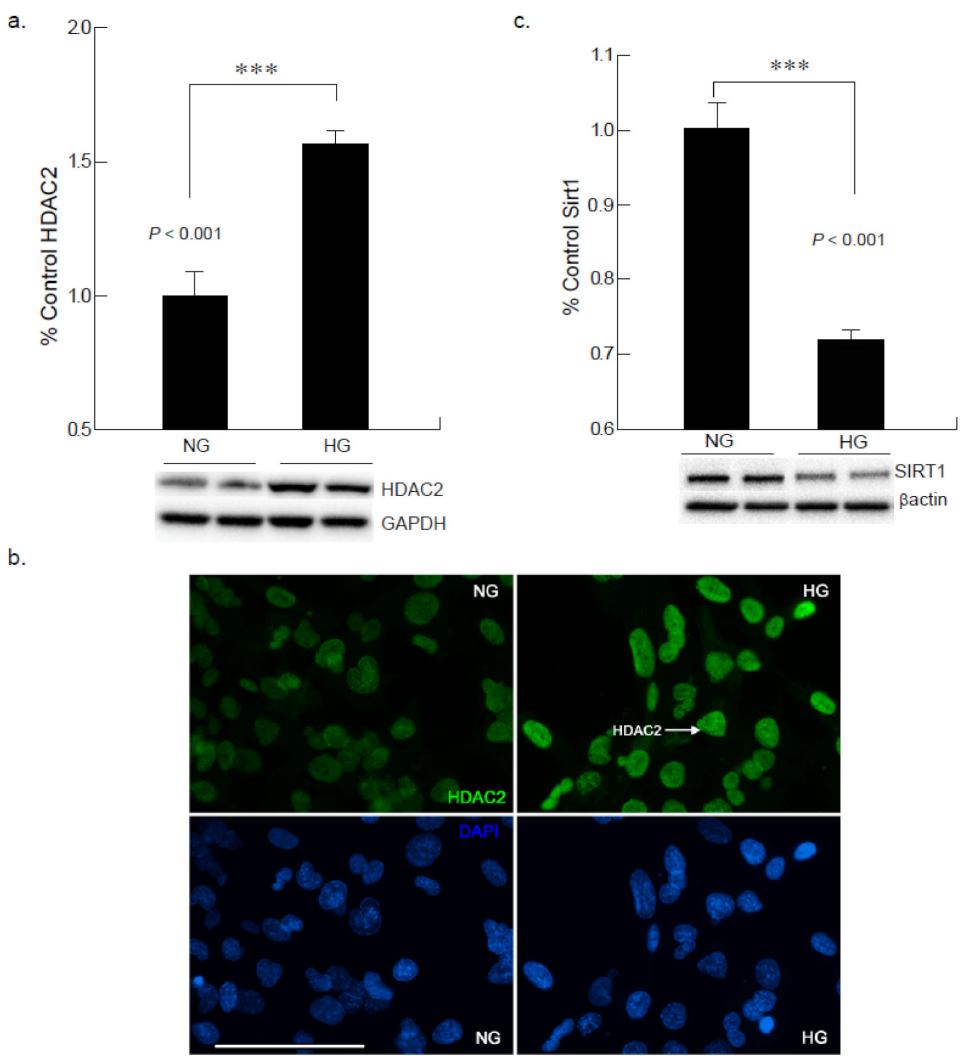

Figure 7. Hyperglycemic exposure exhibits alterations in epigenetic modifications with increased expression of HDAC2 and decreased expression of SIRT1 in cardiomyocytes in vitro. (a) Western blot analysis showed an increase in HDAC2 after $24 \mathrm{~h}$ of exposure to high glucose (HG) in comparison to normal glucose conditions (NG; ${ }^{* * *} p<0.001$ ). (b) Immunocytochemical analysis of cardiomyocytes exposed to high glucose for $24 \mathrm{~h}$ showed an increase in the expression of HDAC2 (green) (Scale bar $=100 \mu \mathrm{m}$ ). (c) Cardiomyocytes exposed to high glucose for $48 \mathrm{~h}$ showed reduction in SIRT1 expression $\left({ }^{* *} p<0.001\right)$.

\section{Discussion}

The purpose of this study was to distinguish if alterations in glucose levels in AC16 cardiomyocyte cells could result in the activation of epigenetic and inflammatory mechanisms that may affect post-translational modification of oxidative stress mediators. Hyperglycemia increased the expression levels of oxidative stress mediators in AC16 cardiomyocytes after 24- and 48-h of exposure to high glucose.

Connexin 43 (Cx43), a gap junction protein, located between cardiomyocyte cells was noted to be down-regulated in the presence of hyperglycemia in the present study. The development and progression of diabetic cardiomyopathy is associated with gap junction activity for intercellular communication [16]. The effect of hyperglycemic conditions on the expression of cardiomyocyte-specific connexin 43 was examined to delineate the activity of the primary gap junction protein for intercellular communication. The results reveal that high glucose concentrations inhibited gap junction activity by reducing $C \times 43$ synthesis in AC16 cardiomyocytes. Degradation of Cx43 will decrease the gap junction activity which may also decrease the conductivity in the cardiomyocytes. Impaired intercellular communication may impact to further changes in cardiac damage markers [17]. In conjunction to this, increased Troponin I expression seen in the AC16 cell line further promotes the concept that the cardiomyocytes may demonstrate injury which is secondary to chronic hyperglycemic stress. The troponin complex is often used to measure cardiac damage in the presence of prolonged stress [18]. More specifically, Troponin I is released in response to stress-induced permanent damage to the cardiac structure [19]. The alterations in Cx43 and Troponin I expressions as evaluated by western blot and immunocytochemical studies after 
24-h exposure suggest the impaired function of the cardiomyocytes under hyperglycemic stress.

Lastly, it was noted via immunocytochemical analysis that the expression of HMGB1 and histone markers were upregulated, which may demonstrate cardiomyocyte apoptosis. HMGB1 is a stress related mediator that plays a pro-inflammatory role when released [20]. This in turn could lead to upregulated immune responses and in turn initiation of apoptotic pathways as the development of cardiomyopathy under hyperglycemia. H3K9me1 and HDAC2 in turn have the role of silencing transcription of genes. Since both followed an upregulated trend at $24 \mathrm{~h}$, it may be assumed that the transcription of cardiomyocytes is being repressed as a result of the pro-inflammatory mechanisms initiated by HMGB1. Prior research has made it evident that inhibiting HDACs promotes healthy cell development and function, which in turn reduces complications associated with elevated blood glucose levels [21]. SIRT1 activation is induced by increased ionized NAD, and conversely a shift in the NADH/NAD+ ratio, commonly observed in hyperglycemia, decreases SIRT1 expression, potentially leading to detrimental effects in the cell.

In the present study a downregulation in epigenetic marker SIRT1 was apparent in the cells that were exposed to hyperglycemia after 48-h exposure. SIRT1 is an NAD+dependent deacetylase involved in metabolic control [15]. The most probable explanation as to why behind these results could be that under hyperglycemic-induced stress, maintenance of NAD+ is challenged. This, then, reduces the pro-survival effects such as: clearance of toxic aggregates, activation of proteolytic enzymes, enhanced expression of chaperone proteins, and inflammation reduction associated with SIRT1 [22]. This suggests that a crosstalk occurring between epigenetic mechanisms and the inflammatory pathway may show inverse relationship as evident by downregulation of SIRT1 expression [23]. Additionally, there was also evidence for a significant decrease in HSP60 expression and a downward trend for HSP70 expression noted throughout the study [8]. The HSP families are tasked with insuring proper folding of proteins and preventing damaged proteins from continuation of folding [24]. Given that SIRT1 was downregulated after 48-h high glucose exposure, which supports that there is communication between the epigenetic mechanisms and the stress-mediation mechanisms in response to increased oxidative stress [25]. This also substantiates that there is possible crosstalk between the epigenetic mechanisms and inflammatory pathways.

It is important to understand the unescapable role of epigenetic processes that are implicated in the development of diabetes complications. The relationship between microvascular and macrovascular complications of diabetes and epigenetic processes has been commonly investigated [26]. Whereas, epigenetic dysregulation towards cardiac atrophy in diabetes has not been investigated thoroughly. The two most commonly studied epigenetic modifications, DNA methylation and the post-translational modification of histone proteins have shown to play key roles in cardiac development as well as cardiac dysfunction [27].

This study suggests that targeting epigenetic markers and stress-mediated responders may impact future approaches in the treatment of cardiomyopathy that has been developed secondary to hyperglycemia. Understanding the crosstalk between the inflammatory mechanisms and epigenetic markers could help researchers and clinicians design enhanced testing methods which could in turn translate into better detection methods and allow for earlier intervention.

\section{Materials and Methods}

\subsection{AC16 Human Cardiomyocyte Cell Line Culture}

AC16 human cardiomyocyte cell line (MilliporeSigma, St. Louis, MO, USA; cat no. SCC109) were cultured in Dulbecco's Modified Eagle's Medium/Nutrient Mixture F-12 Ham (DME/F-12) supplemented with 10\% fetal bovine serum (FBS). This cell line was originally derived from the fusion of primary adult human ventricular heart tissue cells with SV40 transformed, uridine auxotroph human fibroblasts, lacking mitochondrial DNA. 
Following the use of a uridine-free medium as a selection for the removal of unfused fibroblasts, the remaining fused cells were sub-cloned and screened for the presence of SV40 large T-ag, $\beta$-myosin heavy chain ( $\beta$ MHC) and connexin 43 (Cx43) [28]. The cells were plated with approximately $40 \%$ confluency at $24-\mathrm{h}$ and $30 \%$ confluency for 48 -h experiments in 12-well plates for high glucose exposure assessments. When the cells reached $70-80 \%$ confluency, the wells were divided evenly into a control group containing $5 \mathrm{mM}$ of glucose and an experimental group exposed to an additional $25 \mathrm{mM}$ of glucose for hyperglycemic condition.

\subsection{Protein Extraction and Western Blot Analysis}

Following the experimental high glucose exposure period of 24 and $48 \mathrm{~h}$ respectively, the AC16 cells were harvested using lysis buffer with protease and phosphatase inhibitors and processed for western blot as described in our previous work [29,30]. The primary antibodies for RAGE, CX43, HMGB1, SIRT1 (Cell Signaling, Danvers, MA, USA), CXCR4, Trp-I, TLR4, (Thermo Scientific, Waltham, MA, USA), H3K9me1 (Epigentek, Farmingdale, NY, USA), HSP60 (MilliporeSigma, St. Louis, MO, USA) and secondary antibody anti-rabbit IgG or anti-mouse IgG (1:5000 Amersham, Piscataway, NJ, USA) were used according to the protocol and imaged with ECL (Pierce, Rockford, IL, USA). $\beta$-actin (1:2000; MilliporeSigma, St. Louis, MO, USA) was used as a loading control and the data were normalized with the respective level of $\beta$-actin using an image analysis software (ChemiDoc XRS System, Bio-Rad Laboratories, Hercules, CA, USA) to determine the intensity of each band ( $n=3-4$ wells per group), the data were further analyzed to assess the percent of control.

\subsection{Immunocytochemistry}

AC16 cells cultured in coverslips, were rinsed in $1 \mathrm{X}$ phosphate buffered saline (PBS) and subsequently fixed in methanol for $15 \mathrm{~min}$. The coverslips were then blocked at room temperature for $60 \mathrm{~min}$, were washed once and incubated with either of the primary antibodies CX43, HMGB1 (1:400; Cell Signaling, Danvers, MA, USA) or CXCR4, Troponin I (1:500; Thermo-Fisher Scientific, Waltham, MA, USA), H3K9me1 (Epigentek, Farmingdale, NY, USA), for overnight at $4{ }^{\circ} \mathrm{C}$ followed by three washes. Alexa Fluor 594 goat antirabbit IgG and 488 goat anti-mouse IgG (1:2000; Thermo-Fisher Scientific, Waltham, MA, USA) used as secondary antibodies and were incubated for one hour at room temperature. The cells were rinsed in 1X PBS for 3 times and stained with Hoechst solution $(1: 50,000)$. The coverslips were rinsed in 1X PBS for three 5-min intervals, mounted on glass slides using of Fluoromount $\mathrm{G}$ mounting media (Electron Microscopy Sciences, Fort Washington, PA, USA). CM grown on coverslips were immunostained with HMGB1, H3K9me1, CXCR4, CX-43 or Trp-I and imaged to evaluate for changes in intensity to identify the effects of the hyperglycemia. The images were acquired with 20X objective using a Nikon Eclipse Ni-E microscope (Nikon Instruments Inc., Melville, NY, USA).

\subsection{Statistical Analysis}

Normoglycemic and hyperglycemic samples were evaluated to analyze the changes in protein expression. A one-way analysis of variance (ANOVA, parametric) was conducted for the group comparisons using the Bonferroni correction for multiple post hoc analyses. All the statistical analysis was performed using SPSS software (Systat version 13.0, SPSS Inc., Chicago, IL, USA), and a $p$-value $\leq 0.05$ was considered to be statistically significant. The results were demonstrated as mean \pm SEM.

Supplementary Materials: The following are available online at https:/ / www.mdpi.com/article/10 .3390/ijms221910802/s1. FigureS1. Hyperglycemic insult in cardiomyocytes reduces HS70 expression. (a) Western blot analysis of AC16 cardiomyocytes following hyperglycemic insult for 48 hours showed a decrease in the expression of HSP70 as compared to cells exposed to normal glucose $\left(\mathrm{NG}^{*} p<0.05\right)$.

Author Contributions: V.T. and M.C. designed the study. V.T., N.A. and J.C. conducted the experiments and collected data. All authors were involved in analysis and interpretation of the data, 
drafting and finalizing the manuscript. M.C. supervised the study and wrote the manuscript. All authors have read and agreed to the published version of the manuscript.

Funding: This work was supported by the funding from the National Science Foundation; Award No.1949909.

Institutional Review Board Statement: Not applicable.

Informed Consent Statement: Not applicable.

Data Availability Statement: All data generated or analyzed during the current study are included in this published article.

Conflicts of Interest: The authors declare no competing interests.

$\begin{array}{ll}\text { Abbreviations } & \\ \text { CXCR4 } & \text { chemokine C-X-C motif receptor } 4 \\ \text { RAGE } & \text { receptor for advanced glycation end products } \\ \text { Toll-like receptor } 4 & \text { TLR4 } \\ \text { CX43 } & \text { Connexin-43 } \\ \text { Trp-I: } & \text { Troponin I } \\ \text { Sirtuin } 1 & \text { SIRT1 } \\ \text { Histone deacetylase } 2 & \text { HDAC2 } \\ \text { Heat shock protein } 60 & \text { HSP60 } \\ \text { Histone 3 lysine } 9 \text { mono-methylation } & \text { H3K9me1 } \\ \text { High mobility group box } 1 & \text { HMGB1 }\end{array}$

\section{References}

1. Braunwald, E. Cardiomyopathies: An Overview. Circ. Res. 2017, 121, 711-721. [CrossRef]

2. Zhou, P.; Pu, W.T. Recounting Cardiac Cellular Composition. Circ. Res. 2016, 118, 368-370. [CrossRef] [PubMed]

3. Sharma, S.; Jackson, P.G.; Makan, J. Cardiac troponins. J. Clin. Pathol. 2004, 57, 1025-1026. [CrossRef] [PubMed]

4. Yao, D.; Brownlee, M. Hyperglycemia-induced reactive oxygen species increase expression of the receptor for advanced glycation end products (RAGE) and RAGE ligands. Diabetes 2010, 59, 249-255. [CrossRef]

5. Tsan, M.F.; Gao, B. Endogenous ligands of Toll-like receptors. J. Leukoc. Biol. 2004, 76, 514-519. [CrossRef]

6. Tobias, P.S.; Curtiss, L.K. Toll-like receptors in atherosclerosis. Biochem. Soc. Trans. 2007, 35, 1453-1455. [CrossRef]

7. Willis, M.S.; Patterson, C. Hold me tight: Role of the heat shock protein family of chaperones in cardiac disease. Circulation 2010, 122, 1740-1751. [CrossRef] [PubMed]

8. $\quad$ Chen, H.S.; Shan, Y.X.; Yang, T.L.; Lin, H.D.; Chen, J.W.; Lin, S.J.; Wang, P.H. Insulin deficiency downregulated heat shock protein 60 and IGF-1 receptor signaling in diabetic myocardium. Diabetes 2005, 54, 175-181. [CrossRef]

9. Schafler, A.E.; Kirmanoglou, K.; Balbach, J.; Pecher, P.; Hannekum, A.; Schumacher, B. The expression of heat shock protein 60 in myocardium of patients with chronic atrial fibrillation. Basic Res. Cardiol. 2002, 97, 258-261. [CrossRef]

10. Yerra, V.G.; Advani, A. Histones and heart failure in diabetes. Cell. Mol. Life Sci. 2018, 75, 3193-3213. [CrossRef]

11. Yu, X.Y.; Geng, Y.J.; Liang, J.L.; Lin, Q.X.; Lin, S.G.; Zhang, S.; Li, Y. High levels of glucose induce apoptosis in cardiomyocyte via epigenetic regulation of the insulin-like growth factor receptor. Exp. Cell Res. 2010, 316, 2903-2909. [CrossRef]

12. Weeks, K.L.; Avkiran, M. Roles and post-translational regulation of cardiac class IIa histone deacetylase isoforms. J. Physiol. 2015, 593, 1785-1797. [CrossRef]

13. Boutant, M.; Canto, C. SIRT1 metabolic actions: Integrating recent advances from mouse models. Mol. Metab. 2014, 3, 5-18. [CrossRef] [PubMed]

14. Canto, C.; Gerhart-Hines, Z.; Feige, J.N.; Lagouge, M.; Noriega, L.; Milne, J.C.; Elliott, P.J.; Puigserver, P.; Auwerx, J. AMPK regulates energy expenditure by modulating NAD+ metabolism and SIRT1 activity. Nature 2009, 458, 1056-1060. [CrossRef]

15. Canto, C.; Auwerx, J. Interference between PARPs and SIRT1: A novel approach to healthy ageing? Aging 2011, 3, 543-547. [CrossRef] [PubMed]

16. Sheu, J.J.; Chang, L.T.; Chiang, C.H.; Sun, C.K.; Chang, N.K.; Youssef, A.A.; Wu, C.J.; Lee, F.Y.; Yip, H.K. Impact of diabetes on cardiomyocyte apoptosis and connexin 43 gap junction integrity: Role of pharmacological modulation. Int. Heart J. 2007, 48, 233-245. [CrossRef]

17. Tirziu, D.; Giordano, F.J.; Simons, M. Cell communications in the heart. Circulation 2010, 122, 928-937. [CrossRef]

18. Zheng, J.; Ye, P.; Luo, L.; Xiao, W.; Xu, R.; Wu, H. Association between blood glucose levels and high-sensitivity cardiac troponin $\mathrm{T}$ in an overt cardiovascular disease-free community-based study. Diabetes Res. Clin. Pract. 2012, 97, 139-145. [CrossRef]

19. Rubin, J.; Matsushita, K.; Ballantyne, C.M.; Hoogeveen, R.; Coresh, J.; Selvin, E. Chronic hyperglycemia and subclinical myocardial injury. J. Am. Coll. Cardiol. 2012, 59, 484-489. [CrossRef] 
20. Chen, Q.; Guan, X.; Zuo, X.; Wang, J.; Yin, W. The role of high mobility group box 1 (HMGB1) in the pathogenesis of kidney diseases. Acta Pharm. Sin. B 2016, 6, 183-188. [CrossRef] [PubMed]

21. Christensen, D.P.; Dahllof, M.; Lundh, M.; Rasmussen, D.N.; Nielsen, M.D.; Billestrup, N.; Grunnet, L.G.; Mandrup-Poulsen, T. Histone deacetylase (HDAC) inhibition as a novel treatment for diabetes mellitus. Mol. Med. 2011, 17, 378-390. [CrossRef]

22. Ng, F.; Tang, B.L. When is Sirt1 activity bad for dying neurons? Front. Cell. Neurosci. 2013, 7, 186. [CrossRef]

23. Wang, S.; Wang, J.; Zhao, A.; Li, J. SIRT1 activation inhibits hyperglycemia-induced apoptosis by reducing oxidative stress and mitochondrial dysfunction in human endothelial cells. Mol. Med. Rep. 2017, 16, 3331-3338. [CrossRef]

24. Liyanagamage, D.; Martinus, R.D. Role of Mitochondrial Stress Protein HSP60 in Diabetes-Induced Neuroinflammation. Mediators Inflamm. 2020, 2020, 8073516. [CrossRef] [PubMed]

25. Wang, A.J.; Wang, S.; Wang, B.J.; Xiao, M.; Guo, Y.; Tang, Y.; Zhang, J.; Gu, J. Epigenetic Regulation Associated With Sirtuin 1 in Complications of Diabetes Mellitus. Front. Endocrinol. 2020, 11, 598012. [CrossRef]

26. Hussain, S.; Khan, A.W.; Akhmedov, A.; Suades, R.; Costantino, S.; Paneni, F.; Caidahl, K.; Mohammed, S.A.; Hage, C.; Gkolfos, C.; et al. Hyperglycemia Induces Myocardial Dysfunction via Epigenetic Regulation of JunD. Circ. Res. 2020, 127, $1261-1273$. [CrossRef] [PubMed]

27. Yin, L.; Chilian, W.M.; Dong, F. Epigenetic regulation in diabetes-associated oxidative stress and myocardial dysfunction. Int. J. Cardiol. 2018, 268, 193-194. [CrossRef] [PubMed]

28. Davidson, M.M.; Nesti, C.; Palenzuela, L.; Walker, W.F.; Hernandez, E.; Protas, L.; Hirano, M.; Isaac, N.D. Novel cell lines derived from adult human ventricular cardiomyocytes. J. Mol. Cell Cardiol. 2005, 39, 133-147. [CrossRef]

29. Chattopadhyay, M.; Mata, M.; Fink, D.J. Continuous delta-opioid receptor activation reduces neuronal voltage-gated sodium channel (NaV1.7) levels through activation of protein kinase C in painful diabetic neuropathy. J. Neurosci. 2008, 28, 6652-6658. [CrossRef]

30. Thakur, V.; Alcoreza, N.; Delgado, M.; Joddar, B.; Chattopadhyay, M. Cardioprotective Effect of Glycyrrhizin on Myocardial Remodeling in Diabetic Rats. Biomolecules 2021, 11, 569. [CrossRef] [PubMed] 